\title{
Automatic detection of spermatozoa for laser capture microdissection
}

\author{
Mado Vandewoestyne • David Van Hoofstat • \\ Filip Van Nieuwerburgh • Dieter Deforce
}

Received: 28 December 2007 / Accepted: 19 June 2008 / Published online: 26 July 2008

(C) The Author(s) 2008

\begin{abstract}
In sexual assault crimes, differential extraction of spermatozoa from vaginal swab smears is often ineffective, especially when only a few spermatozoa are present in an overwhelming amount of epithelial cells. Laser capture microdissection (LCM) enables the precise separation of spermatozoa and epithelial cells. However, standard spermstaining techniques are non-specific and rely on sperm morphology for identification. Moreover, manual screening of the microscope slides is time-consuming and laborintensive. Here, we describe an automated screening method to detect spermatozoa stained with Sperm HYLITER $^{\text {TM }}$. Different ratios of spermatozoa and epithelial cells were used to assess the automatic detection method. In addition, real postcoital samples were also screened. Detected spermatozoa were isolated using LCM and DNA analysis was performed. Robust DNA profiles without allelic dropout could be obtained from as little as 30 spermatozoa recovered from postcoital samples, showing that the staining had no significant influence on DNA recovery.
\end{abstract}

Keywords Forensic science - Sexual assault . Laser capture microdissection $\cdot$ Spermatozoa .

Automatic cell recognition

\section{Introduction}

In cases of sexual assault, DNA typing, using biological material such as vaginal smears is a common method for

M. Vandewoestyne · D. Van Hoofstat · F. Van Nieuwerburgh •

D. Deforce $(\square)$

Laboratory for Pharmaceutical Biotechnology, Ghent University,

Harelbekestraat 72,

9000 Ghent, Belgium

e-mail: Dieter.Deforce@Ugent.be suspect identification. The spermatozoa are traditionally separated from the vaginal epithelial cells by differential extraction. The epithelial cells are preferentially lysed by incubation in an SDS/proteinase $\mathrm{K}$ mixture, while spermatozoa are resistant to this treatment and can be separated by centrifugation [1]. The remaining supernatant containing the victim's DNA is removed and the spermatozoa can be lysed in a buffer containing dithiothreitol (DTT). However, a certain amount of vaginal cells remain undigested during the initial steps so that if there are only a few spermatozoa on the microscope slide, the DNA of the perpetrator is contaminated by the victim's DNA, resulting in a mixed DNA profile [2] hereby making this method ineffective. In some cases the profile of the perpetrator cannot be detected due to the large amount of victim's DNA.

Another option is the use of Y-chromosome short tandem repeats (Y STR) to detect the male component in mixed stains when the DNA of the male contributor is present in a small amount [3]. Since the discrimination power of Y STR analysis is much lower than autosomal STR analysis, it is only of interest in cases where no autosomal DNA profile of the semen contributor could be detected.

The use of laser capture microdissection (LCM) has been evaluated for the isolation of spermatozoa from microscope slides containing both spermatozoa and vaginal cells [4-6]. Elliot et al. showed that LCM outperforms differential extraction for the recovery of DNA from sperm cells, especially in cases where only a few sperm cells are present [7]. LCM was also used for the isolation of male cells in a male/female cell mixture $[8,9]$.

The search for spermatozoa on a microscope slide by visual inspection can often be very time-consuming and labor-intensive, especially for slides containing a low number of spermatozoa. Standard methods to identify 
spermatozoa are based on non-specific staining techniques such as hematoxylin/eosin (HE) and nuclear fast red/ picroindigocarmine (CTS or "Christmas tree stain") [10], which rely on sperm morphology for identification [4] and cannot be used for automated sperm detection. Moreover HE staining reduces the yield of DNA $[4,5,11]$ and CTS staining results in DNA damage, probably due to the picric acid component [4].

Sperm HY-LITER ${ }^{\mathrm{TM}}$ (Independent Forensics, Hillside, IL, USA) is a fluorescent kit for the detection of human spermatozoa which does not rely on morphological characteristics or non-specific staining for identification. All relevant information concerning this kit can be found at http://www. spermhy-liter.com/. The spermatozoa are detected using an Alexa Fluor 488 derivatized mouse monoclonal antibody against proteins contained in the human sperm heads. In addition, a 4',6-diamidino-2-phenylindole staining is used to detect all nuclei present on the slide. Processed slides can be viewed at low magnification, greatly increasing the speed of sperm identification.

The PALM MicroBeam system (P.A.L.M. Microlaser Technologies, Bernried, Germany), allows scanning of microscope slides [5]. The system can be supplemented with image analyzing software modules allowing automated specimen identification and image processing [12] e.g. the AxioVision Commander (Carl Zeiss, Hallbergmoos, Germany). This image analyzing software can be optimized to automatically detect cells with specific features. In the current report we have optimized for the automatic detection of spermatozoa, stained with Sperm HY-LITER ${ }^{\text {TM }}$ (Independent Forensics). The detected spermatozoa can then be collected by a defined laser pulse using the PALM laser pressure catapulting (LPC) function. This contact-free cell collection is an ideal approach to avoid sample contamination in forensic cases.

In the present study, an automated scanning method was developed to identify sperm heads, stained with Sperm HYLITER $^{\mathrm{TM}}$ (Independent Forensics), on smear preparations from postcoital vaginal swabs. LPC was used to catapult the auto-detected spermatozoa and DNA analysis was performed. After optimization of the DNA extraction protocol, the effect of the Sperm HY-LITER ${ }^{\mathrm{TM}}$ staining on downstream analysis of laser pressure catapulted spermatozoa was examined and the ability to obtain DNA profiles from spermatozoa catapulted from stained postcoital vaginal smears was assessed.

\section{Materials and methods}

Sample preparation

Vaginal epithelial cells from healthy volunteers were collected onto sterile cotton swabs. These swabs were agitated in phosphate buffered saline (PBS, Gibco, Paisly, UK). After cell counting, PBS was added to obtain an epithelial cell working solution of $2 \times 10^{6}$ cells $/ \mathrm{ml}$. Semen samples were obtained from healthy volunteers. Semen working solutions of $2 \times 10^{6}$ spermatozoa/ml were prepared by diluting the liquid semen samples in PBS. Mixtures were prepared by combining semen working solution with epithelial cell working solution in the following ratios: $1: 2$, 1:10, and 1:50. Of each mixture $30 \mu \mathrm{l}$ was used to make smear preparations on routine glass object slides (Dako silanized slides, Glostrup, Denmark). In addition, pure semen smears and postcoital vaginal swab smears were made.

The preparations were all dried overnight at room temperature and stained using the Sperm HY-LITERTM kit (Independent Forensics) according to manufacturer's suggested protocol with slight modifications: the preparations were fixed for 2 min using two drops of a $70 \%$ ethanol solution (absolute ethanol, Merck BV, Schiphol-Rijk, The Netherlands) in pure water (MilliQ, Millipore, Billerica, MA, USA) in stead of the Sperm HY-LITER ${ }^{\text {TM }}$ fixation solution. After the last washing step in the protocol, the slides were washed with $2 \mathrm{ml}$ of pure water (MilliQ, Millipore) to remove adherent salts from the Sperm HYLITER $^{\text {TM }}$ wash buffer and the stained slides were visualized immediately without mounting.

\section{Fluorescence scanning}

The scanning stage was controlled by the PALM RoboSoftware version 4 (P.A.L.M. Microlaser Technologies). Image acquisition was carried out with the AxioVision multichannel fluorescence module (Carl Zeiss) and the AxioCam MRm camera (Carl Zeiss).

The cell nuclei, including epithelial and sperm cell nuclei, were visualized using Zeiss filter set no. 49 (G 365 nm, FT 495, BP 445/50). The spermatozoa were visualized using Zeiss filter set no. 38 (BP 470/40, FT 495, BP 525/50). The slides were scanned at $20 \times$ magnification using a Carl Zeiss long distance Plan-Neofluar ${ }^{\circledR}$ objective. From every slide, 100 images were acquired using the scanning mode. The acquired images were displayed as an overview image in the PALM Navigator window and the individual images were stored as tiff-files.

Segmentation and masking

For automated detection of spermatozoa, the image processing AxioVision Commander module (Carl Zeiss) was used. All steps of processing, analysis, and evaluation were stored in an AxioVision Commander Script, which could be run automatically on the stored images.

In a first step the sperm heads, detected in the green fluorescence channel, were discriminated from the back- 
ground using an interactive threshold. The resulting image was a binary image where the background was black and the detected regions were white. In a following step all detected regions below $3 \mu \mathrm{m}$ or above $7 \mu \mathrm{m}$ were removed from the binary image to eliminate artefacts smaller or bigger than a sperm head (approximately $5 \mu \mathrm{m}$ ).

Then the nuclei detected in the blue fluorescence channel were distinguished from the background using an interactive threshold. Afterwards artefacts (regions with an area above $70 \mu^{2}$ ) were also eliminated from the resulting binary image. Following this procedure all nuclei still touching each other were separated using a watershed algorithm, to make sure every nucleus was counted separately. This algorithm splits the image into disjoint regions containing only one nucleus and is based on the topology of the image.

In a next step the spermatozoa were identified by masking the binary image of the green channel and the binary image of the blue channel, as shown in Fig. 1. Only regions of the binary image of the nuclei which were overlaid by at least one pixel of the binary image of the sperm heads were kept in the resulting image. In this way, remaining artefacts with the same dimensions as a sperm head but without a nucleus were removed from the resulting image.

The coordinates of the detected spermatozoa were transferred automatically to an element list in the PALM
RoboSoftware and after visual verification of the detected spermatozoa a catapulting point was set on the sperm head.

\section{Laser pressure catapulting}

The detected spermatozoa were collected by laser pressure catapulting using a pulsed nitrogen UV-A laser (wavelength $355 \mathrm{~nm}$ ). The high energy generated by the focused laser light was used to catapult the detected spermatozoa into the cap of a standard $0.2 \mathrm{ml}$ microfuge tube (Westburg, Leusden, The Netherlands) containing $18 \mu$ l of PicoPure DNA extraction buffer (PicoPure DNA extraction kit, Arcturus, Mountain View, CA, USA) supplemented with $2 \mu \mathrm{l}$ of 1 M DTT (MP Biomedicals, Solon, OH, USA). This contact-free method avoids contamination of the sample.

After the catapulting process, the recovery of the spermatozoa could be verified microscopically in the collection caps. In addition, as a verification of the catapulting process, fluorescent images were acquired before and after LPC, as shown in Fig. 2.

\section{DNA extraction}

DNA was extracted from the catapulted spermatozoa, using the PicoPure DNA extraction kit (Arcturus). The samples
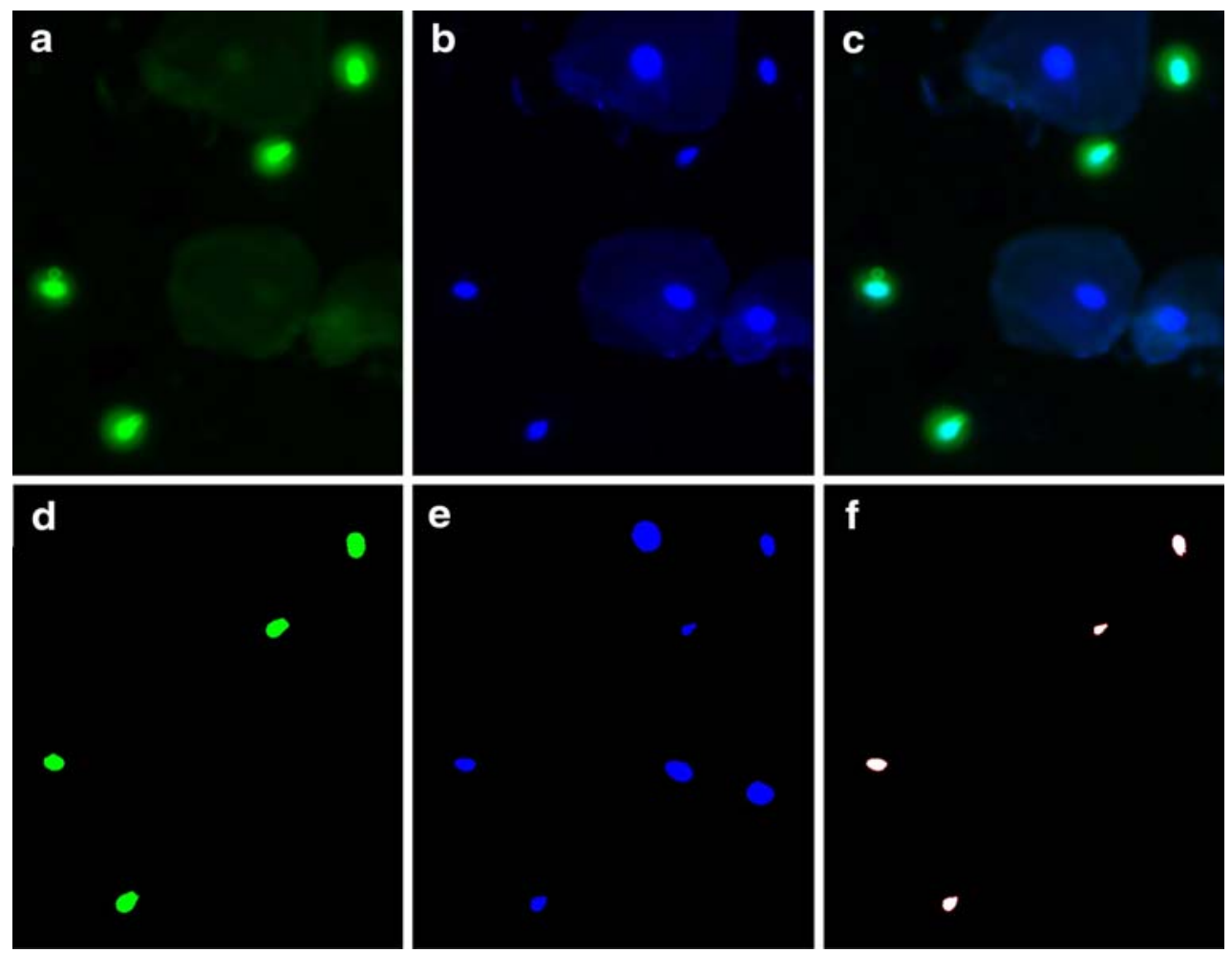

Fig. 1 Principle of the AxioVision Commander Script. a Pseudocolored original image of the sperm heads, $\mathbf{b}$ pseudo-colored original image of the nuclei, $\mathbf{c}$ overlay of both pseudo-colored images, $\mathbf{d}$ binary image of the sperm heads after segmentation, e binary image of the nuclei after segmentation, $\mathbf{f}$ final masked image outlining the detected sperm heads 

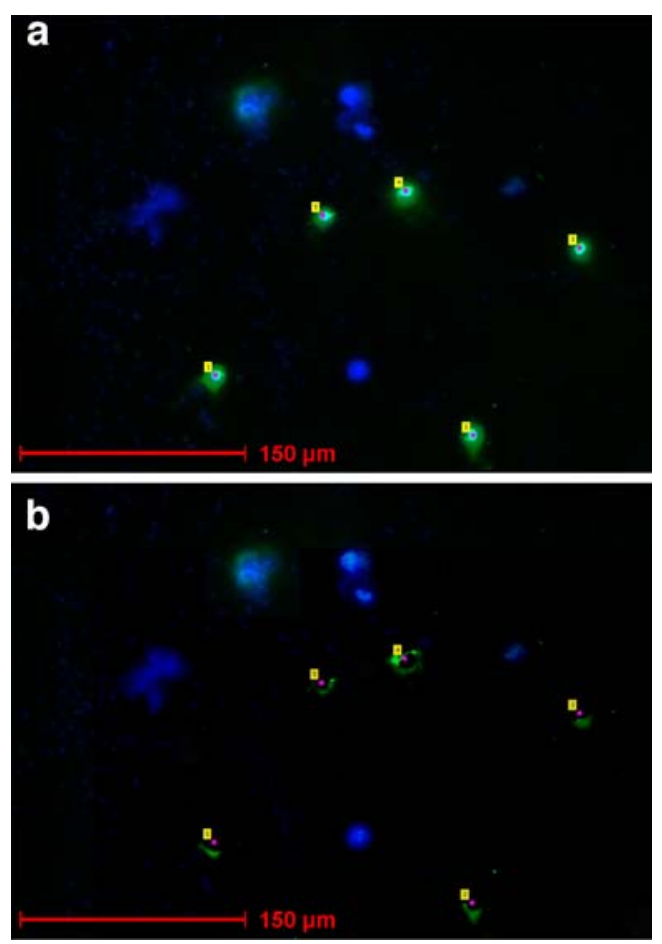

Fig. 2 Laser pressure catapulting of detected spermatozoa. Same field of view: a fluorescent image before laser pressure catapulting, b fluorescent image after laser pressure catapulting

were incubated at $65^{\circ} \mathrm{C}$ overnight, centrifuged briefly and heated to $95^{\circ} \mathrm{C}$ for $30 \mathrm{~min}$ to inactivate proteinase $\mathrm{K}$. If the polymerase chain reaction (PCR) could not be performed immediately, the samples were stored at $-20^{\circ} \mathrm{C}$.

\section{Amplification and detection}

For assessing the profile recovery after LPC from pure semen samples, a multiplex of four STR loci (CD4, TH01, D21S11, and SE33) was used as described earlier [13] with slight modifications. In short, 1.3 units of hotstar Taq DNA polymerase (Qiagen, Huntsville, AL, USA) was used and the samples were amplified on an Applied Biosystems GeneAmp 970060 Well thermal cycler (Foster City, CA, USA). Amplification parameters were: preincubation at $95^{\circ} \mathrm{C}$ for $15 \mathrm{~min}$, followed by 33 cycles of denaturation for $60 \mathrm{~s}$ at $94^{\circ} \mathrm{C}$, annealing for $60 \mathrm{~s}$ at $58^{\circ} \mathrm{C}$ and extension for $80 \mathrm{~s}$ at $72^{\circ} \mathrm{C}$. This was followed by a final elongation step of $5 \mathrm{~min}$ at $72^{\circ} \mathrm{C}$. At the end of the PCR reaction the temperature was kept at $4^{\circ} \mathrm{C}$.

For the amplification of the DNA extracted from the spermatozoa isolated by LPC from the postcoital samples, the AmpF $/ \mathrm{STR}^{\circledR}$ Profiler Plus ${ }^{\circledR}$ kit from Applied Biosystems was used according to the manufacturer's instructions, except that Hotstar Taq DNA polymerase was used instead of Taq Gold. The total number of cycles was 33 .
The amplified fragments were then separated and analyzed by capillary electrophoresis using an ABI 3100 Genetic Analyzer (Applied Biosystems).

\section{Results}

Automated detection of spermatozoa

As the Sperm HY-LITER ${ }^{\text {TM }}$ kit (Independent Forensics) has been designed for microscopic screening of mounted slides, a few modifications of the manufacturer's suggested protocol had to be carried out to make it compatible with
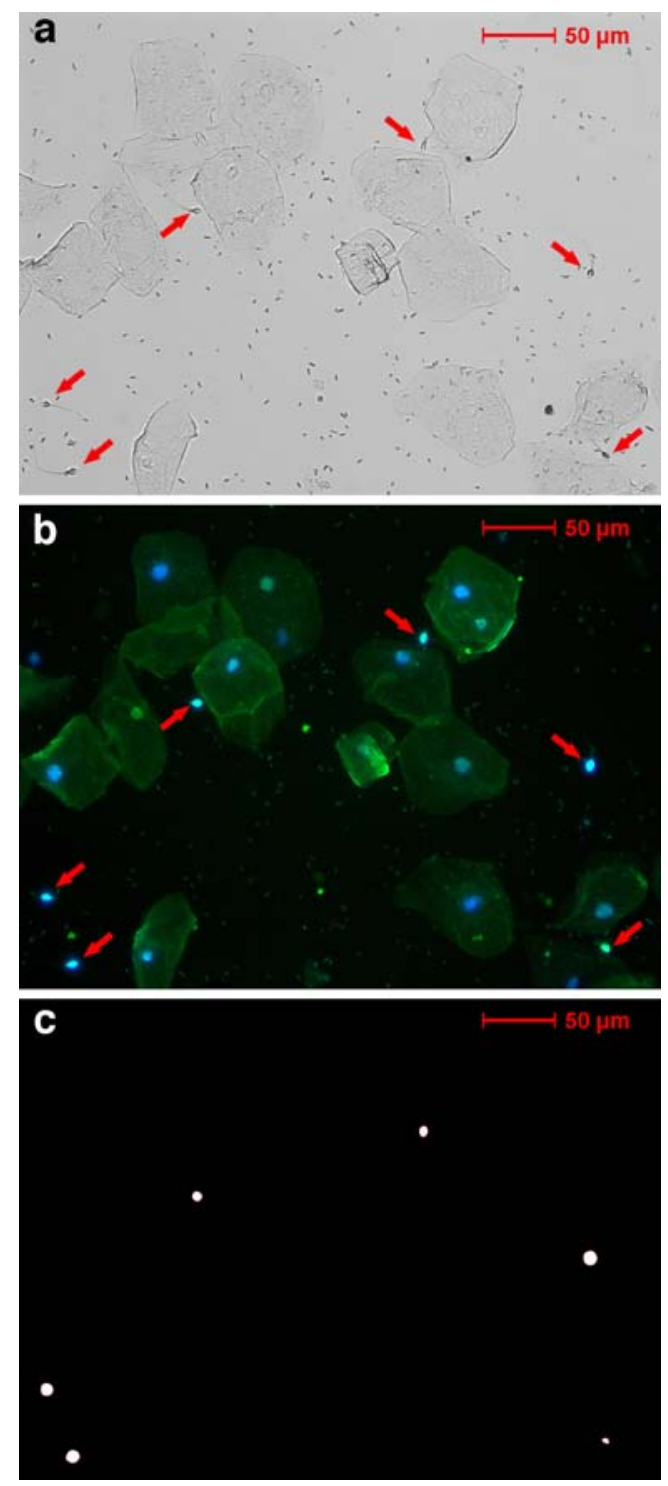

Fig. 3 Automatic detection of spermatozoa by the AxioVision Commander Script. Same field of view: a bright field image, containing six spermatozoa, vaginal epithelial cells and Lactobacillus organisms, b fluorescent image, $\mathbf{c}$ resulting binary image, outlining the six sperm heads 


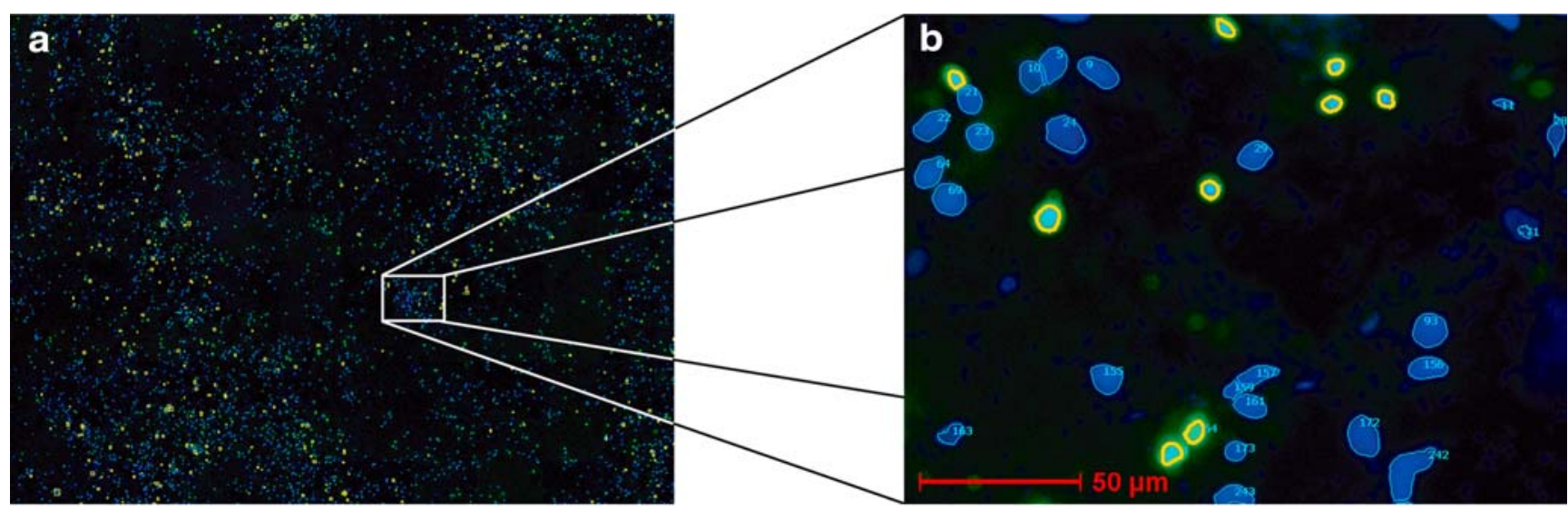

Fig. 4 Automatic scanning of the microscope slide and result of the AxioVision Commander Script. a Overview image of the 100 images acquired from the postcoital vaginal smear. b Zoomed in image $(100 \times$

zoom): vaginal epithelial cells are outlined in light blue, sperm heads are outlined in yellow

ratio, show the very high reliability of the developed AxioVision Commander Script.

The detected spermatozoa were catapulted by LPC in to a standard microfuge tube. Images acquired before and after catapulting clearly show that the catapulting process removes the sperm head from the glass slide.

Profile recovery after laser pressure catapulting

Three DNA isolation methods were evaluated (data not shown): an alkaline extraction method [14], DNA IQ ${ }^{\mathrm{TM}}$ System (Promega Corporation, Madison, WI, USA) and PicoPure DNA extraction kit (Arcturus) and the best results were obtained with the latter. After optimization, this DNA extraction method was used for further assessment of profile recovery after LPC.

Different amounts of spermatozoa $(200,100,75,50,40$, 30,20 , and 10) were collected by LPC from the pure sperm smears to assess the effect of the Sperm HY-LITER ${ }^{\mathrm{TM}}$ staining on the recovery of DNA. A full DNA profile of the four assessed loci (CD4, TH01, D21S11, and SE33) could be recovered from every sample. This proves that the Sperm HY-LITER ${ }^{\mathrm{TM}}$ staining has no negative influence on the DNA quality of the spermatozoa.

In addition, profile recovery from spermatozoa isolated by LPC from postcoital samples was analyzed using the AmpF $/ \mathrm{STR}^{\circledR}$ Profiler Plus ${ }^{\circledR}$ kit from Applied Biosystems.

Table 1 Automatically detected spermatozoa and nuclei in 100 acquired images per slide

\begin{tabular}{lccc}
\hline $\begin{array}{l}\text { Ratio of spermatozoa/nuclei } \\
\text { used to make the smears }\end{array}$ & $\begin{array}{l}\text { Number of sperm } \\
\text { heads detected }\end{array}$ & $\begin{array}{l}\text { Total number of nuclei (spermatozoa } \\
\text { and epithelial cells) detected }\end{array}$ & $\begin{array}{l}\text { Detected ratio of } \\
\text { spermatozoa/nuclei }\end{array}$ \\
\hline $1: 2$ & 1,379 & 3,625 & $1: 2.6$ \\
$1: 10$ & 407 & 2,959 & $1: 7.3$ \\
$1: 50$ & 69 & 2,948 & $1: 42.7$ \\
Postcoital sample & 963 & 4,784 & $1: 4.97$ \\
\hline
\end{tabular}


Table 2 Profile recovery from spermatozoa after LPC from postcoital samples

\begin{tabular}{lccc}
\hline $\begin{array}{l}\text { Number of isolated } \\
\text { spermatozoa }\end{array}$ & $\begin{array}{l}\text { Number of expected } \\
\text { male alleles }\end{array}$ & $\begin{array}{l}\text { Number of detected } \\
\text { male alleles }\end{array}$ & $\begin{array}{l}\text { Number of contaminating } \\
\text { female alleles }\end{array}$ \\
\hline 10 & 17 & 16 & 0 \\
10 & 17 & 15 & 0 \\
20 & 17 & 15 & 0 \\
20 & 17 & 17 & 0 \\
30 & 17 & 17 & 0 \\
30 & 17 & 17 & 0 \\
50 & 17 & 17 & 0 \\
50 & 17 & 17 & 0 \\
\hline
\end{tabular}

Different amounts of spermatozoa (two times 50, 30, 20, and 10 spermatozoa) were collected. The results are presented in Table 2. The two samples where 10 spermatozoa were collected and one sample where 20 spermatozoa were collected show allelic drop out. This is in agreement with the theoretical expected probability of full allelic representation from this number of haploid cells [15]. Full DNA profiles were recovered when 30 or more spermatozoa
Fig. 5 DNA profile recovery after LPC. Profile derived from a postcoital sample after catapulting 50 spermatozoa

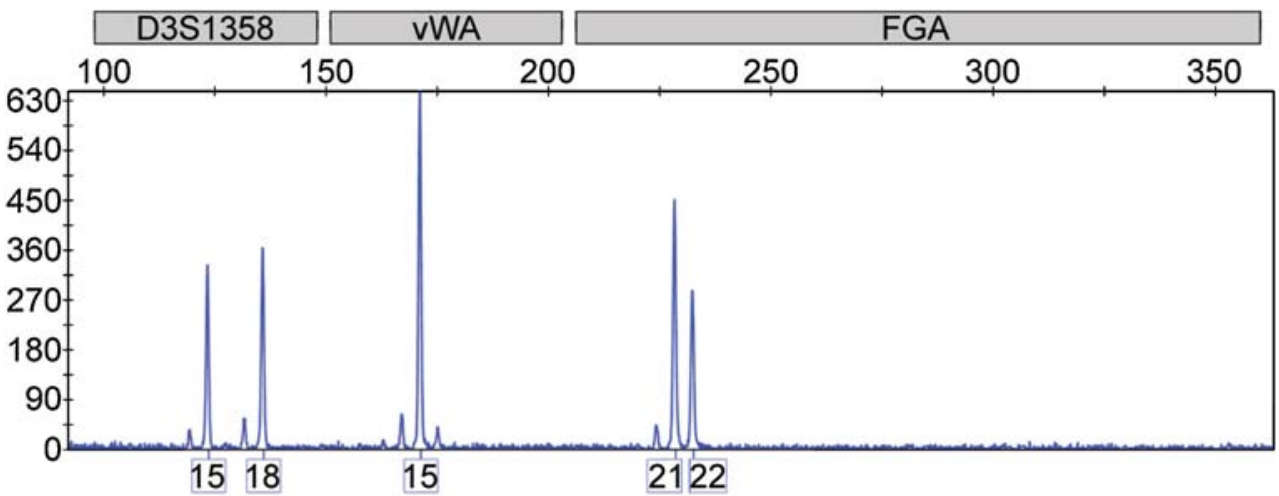

\begin{tabular}{|cc|cccc|}
\hline AM & D8S1179 & D21S11 & & D18S51 \\
\cline { 3 - 5 } 100 & 150 & 200 & 250 & 300 & 350 \\
\hline
\end{tabular}
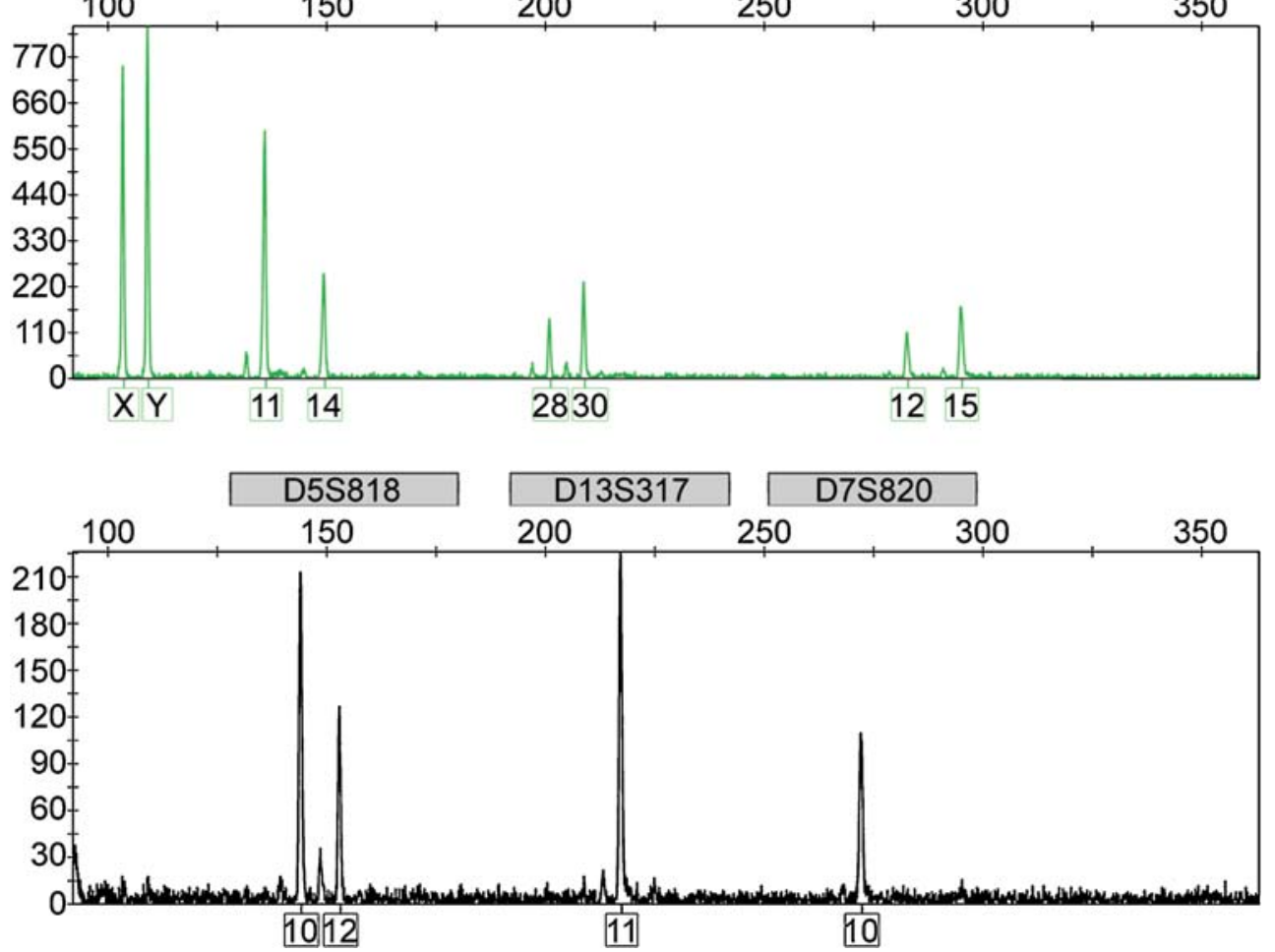
were collected. Figure 5 shows a full DNA profile obtained after catapulting 50 spermatozoa from a postcoital sample. In one of the samples a few extra alleles from the female donor were recovered. This mixture may be due to the adherence of female DNA from lysed epithelial cells to spermatozoa [7, 16]. Despite the presence of female DNA, the male DNA profile was easily interpreted.

\section{Discussion}

The results demonstrate that the AxioVision Commander Script has sufficient sensitivity to identify sperm heads stained with Sperm HY-LITER ${ }^{\text {TM }}$ (Independent Forensics), present in an overwhelming background of epithelial cells. The sperm heads and the cell nuclei are easily detectable and the background staining is negligible. The nucleus staining provides additional confirmation that the Alexa Fluor 488 signal observed is related to a DNA-containing sperm head. The coordinates of the single cells assessed as spermatozoa are transferred to an element list of the PALM RoboSoftware. Before laser catapulting, the detected cells can be verified easily through bright field microscopy based on sperm cell morphology.

The scanning, analysis and identification of spermatozoa can be performed on normal microscopic slides, which are used with the Belgian sexual assault sets, allowing its use in existing preparations from sexual assault cases. Processed slides can be scanned at low magnification, greatly increasing the speed of sperm identification.

For forensic DNA profiling, it is important that the staining reagents used have no adverse effects on the recovery of DNA from spermatozoa on postcoital vaginal swabs. As robust DNA profiles without allelic drop out could be obtained from as little as 30 spermatozoa recovered from postcoital samples after LPC, it can be concluded that the Sperm HY-LITER ${ }^{\mathrm{TM}}$ staining has no influence on downstream DNA analysis, in contrast to traditional nonspecific sperm-staining methods as HE and CTS.

In one of the postcoital samples, female DNA was present in the profile after LPC. This may be due to adherence of female DNA to the spermatozoa $[7,16]$. Despite the presence of female DNA in one of the samples after LPC, the male DNA profile was interpreted easily. Nevertheless, further work will concentrate on generating pure male DNA profiles from this kind of samples.

As the whole procedure of staining, scanning, and catapulting can be performed in half a day, it can be concluded that, used in combination with the automatic detection of spermatozoa by the AxioVision Commander Script, the LCM method is a fast, sensitive and non-contact procedure for DNA profiling in cases of sexual assault.
Acknowledgements The Research was funded by a $\mathrm{PhD}$ grant from the Institute for the Promotion of Innovation through Science and Technology in Flanders (IWT-Vlaanderen) to Mado Vandewoestyne.

The authors are grateful to Wouter Christiaens (Zeiss, Belgium) and Renate Burgemeister \& colleagues (P.A.L.M. Microlaser, Germany) for their help in the development of the AxioVision Commander Script.

Disclaimer The authors declare that they have no competing financial, personal nor professional interests.

Open Access This article is distributed under the terms of the Creative Commons Attribution Noncommercial License which permits any noncommercial use, distribution, and reproduction in any medium, provided the original author(s) and source are credited.

\section{References}

1. Gill P, Jeffreys AJ, Werrett DJ (1985) Forensic application of DNA 'fingerprints'. Nature 318:577-579

2. Wiegand P, Schurenkamp M, Schutte U (1992) DNA extraction from mixtures of body fluid using mild preferential lysis. Int $\mathrm{J}$ Legal Med 104:359-360

3. Cerri N, Ricci U, Sani I, Verzeletti A, De Ferrari F (2003) Mixed stains from sexual assault cases: autosomal or Y-chromosome short tandem repeats? Croat Med J 44:289-292

4. Sanders CT, Sanchez N, Ballantyne J, Peterson DA (2006) Laser microdissection separation of pure spermatozoa from epithelial cells for short tandem repeat analysis. J Forensic Sci 51:748-757

5. Seidl S (2005) Contact-free isolation of sperm and epithelial cells by laser microdissection and pressure catapulting. Forensic Sci Med Pathol 1:153-158

6. Di Martino D, Giuffre G, Staiti N, Simone A, Le Donne M, Saravo L (2004) Single sperm cell isolation by laser microdissection. Forensic Sci Int 146:S151-S153

7. Elliott K, Hill DS, Lambert C, Burroughes TR, Gill P (2003) Use of laser microdissection greatly improves the recovery of DNA from sperm on microscope slides. Forensic Sci Int 137:28-36

8. Anslinger K, Mack B, Bayer B, Rolf B, Eisenmenger W (2005) Digoxigenin labelling and laser capture microdissection of male cells. Int J Legal Med 119:374-377

9. Anslinger K, Bayer B, Mack B, Eisenmenger W (2007) Sexspecific fluorescent labelling of cells for laser microdissection and DNA profiling. Int J Legal Med 121:54-56

10. Allery JP, Telmon N, Mieusset R, Blanc A, Rouge D (2001) Cytological detection of spermatozoa: comparison of three staining methods. J Forensic Sci 46:349-351

11. Ehrig T, Abdulkadir SA, Dintzis SM, Milbrandt J, Watson MA (2001) Quantitative amplification of genomic DNA from histological tissue sections after staining with nuclear dyes and laser capture microdissection. J Mol Diagn 3:22-25

12. Niyaz Y, Sägmüller B (2005) Non-contact laser microdissection and pressure catapulting: automation via object-oriented image processing. Med Laser Appl 20:223-232

13. Van Hoofstat DE, Deforce DL, Millecamps RE et al (1998) Population genetic study of four short tandem repeat loci in the Belgian population, using capillary electrophoresis. Electrophoresis 19:719-722

14. Rudbeck L, Dissing J (1998) Rapid, simple alkaline extraction of human genomic DNA from whole blood, buccal epithelial cells, semen and forensic stains for PCR. Biotechniques 25:588-590 592

15. Lucy D, Curran JM, Pirie AA, Gill P (2007) The probability of achieving full allelic representation for LCN-STR profiling of haploid cells. Sci Justice 47:168-171

16. Spadafora C (1998) Sperm cells and foreign DNA: a controversial relation. Bioessays 20:955-964 